\title{
Novel ionically crosslinked casein nanoparticles for flutamide delivery: formulation, characterization, and in vivo pharmacokinetics
}

This article was published in the following Dove Press journal:

International Journal of Nanomedicine

2 May 2013

Number of times this article has been viewed

\author{
Ahmed O Elzoghby' \\ Maged W Helmy² \\ Wael M Samy ${ }^{1,3}$ \\ Nazik A Elgindy' \\ 'Department of Industrial Pharmacy, \\ Faculty of Pharmacy, Alexandria \\ University, Alexandria, Egypt; \\ ${ }^{2}$ Department of Pharmacology and \\ Toxicology, Faculty of Pharmacy, \\ Pharos University, Alexandria, Egypt; \\ ${ }^{3}$ Department of Pharmaceutics and \\ Pharmaceutical Technology, Faculty \\ of Pharmacy, Beirut Arab University, \\ Beirut, Lebanon
}

\begin{abstract}
A novel particulate delivery matrix based on ionically crosslinked casein (CAS) nanoparticles was developed for controlled release of the poorly soluble anticancer drug flutamide (FLT). Nanoparticles were fabricated via oil-in-water emulsification then stabilized by ionic crosslinking of the positively charged CAS molecules below their isoelectric point, with the polyanionic crosslinker sodium tripolyphosphate. With the optimal preparation conditions, the drug loading and incorporation efficiency achieved were $8.73 \%$ and $64.55 \%$, respectively. The nanoparticles exhibited a spherical shape with a size below $100 \mathrm{~nm}$ and a positive zeta potential $(+7.54$ to $+17.3 \mathrm{mV})$. FLT was molecularly dispersed inside the nanoparticle protein matrix, as revealed by thermal analysis. The biodegradability of CAS nanoparticles in trypsin solution could be easily modulated by varying the sodium tripolyphosphate crosslinking density. A sustained release of FLT from CAS nanoparticles for up to 4 days was observed, depending on the crosslinking density. After intravenous administration of FLT-CAS nanoparticles into rats, CAS nanoparticles exhibited a longer circulation time and a markedly delayed blood clearance of FLT, with the half-life of FLT extended from 0.88 hours to 14.64 hours, compared with drug cosolvent. The results offer a promising method for tailoring biodegradable, drug-loaded CAS nanoparticles as controlled, long-circulating drug delivery systems of hydrophobic anticancer drugs in aqueous vehicles.
\end{abstract}

Keywords: casein nanoparticles, ionic crosslinking, biodegradability, controlled release, in vivo pharmacokinetics

\section{Introduction}

Over the past few decades there has been considerable interest in developing protein nanoparticles as drug delivery vehicles. ${ }^{1,2}$ The underlying rationale is their exceptional characteristics, namely biodegradability, nonantigenicity, abundant renewable sources, extraordinary binding capacity with various drugs, and the ease of scaling up during manufacture. ${ }^{1,2}$ Casein (CAS), a generally recognized as safe protein, is the major milk protein that forms an integral part of the daily diet. It possesses a number of interesting properties that make it a good candidate for conventional and novel drug delivery systems. ${ }^{3,4} \mathrm{CAS}$ may be advantageous as an alternative to albumin as a matrix for drug delivery, because it is inexpensive and has better amphiphilicity, good dispersibility, and rapid reconstitution in aqueous systems. ${ }^{3,4}$

CASs are amphiphilic proteins that can be thought of as block copolymers with high levels of hydrophobic or hydrophilic amino acid residues. Therefore, CASs exhibit a strong tendency to self-assemble into spherical micelles $(50-500 \mathrm{~nm}$ in diameter, $150 \mathrm{~nm}$ average diameter). ${ }^{3,4}$ Only recently, CAS micelles were harnessed for
Correspondence: Ahmed O Elzoghby Department of Industrial Pharmacy, Faculty of Pharmacy, Alexandria University, I Khartoum Square, Azarita, Messalla, PO Box 21521, Alexandria, Egypt

$\mathrm{Tel}+2033825212$

$\mathrm{Fax}+2034873273$

Email dr_ahmedelzoghby@yahoo.com 
delivering exogenous hydrophobic bioactives. CAS micelles effectively protected vitamin $\mathrm{D}_{2}$ and docosahexaenoic acid against ultraviolet (UV) light-induced degradation and oxidation, respectively. ${ }^{5,6}$ In another study, the complexation of curcumin with $\beta$-CAS micelles increased the solubility of curcumin at least 2500 -fold with enhanced curcumin cytotoxicity to a human leukemia cell line. ${ }^{7}$ Shapira et al ${ }^{8,9}$ showed that $\beta$-CAS micelles could entrap and deliver hydrophobic chemotherapeutics such as mitoxantrone and paclitaxel, allowing them to be thermodynamically stable in aqueous solutions for oral delivery applications. Recently, Bachar et $\mathrm{al}^{10}$ successfully developed celecoxib-loaded $\beta$-CAS micelles with high encapsulation loads for oral delivery.

A desolvation technique has been widely used to prepare protein nanoparticles such as albumin and gelatin, and successfully achieved sustained drug release for prolonged periods of time. ${ }^{2,11}$ However, this technique has two major drawbacks: the use of organic solvents, and toxic crosslinking chemicals such as glutaraldehyde. Thus, the clinical application of the resulting nanoparticles is hampered by safety concerns. Therefore, the use of nontoxic crosslinking agents (eg, genipin and transglutaminase) emerged as an alternative to glutaraldehyde. ${ }^{2}$ In our previous study we used a spraydrying technique to generate genipin-crosslinked CAS nanoparticles for prolonged release of alfuzosin hydrochloride. ${ }^{12}$

Prostate cancer has the highest incidence of all kinds of cancers and is the second most deadly cancer in men, after lung cancer. ${ }^{13}$ Flutamide (FLT) is an antiandrogenic agent presently used for monotherapy of androgen-dependent prostate cancer. ${ }^{14}$ It acts by inhibiting the uptake and/or binding of dihydrotestosterone to the target cell receptor, thus interfering with androgen action, which requires stability in blood for a sufficient amount of time. ${ }^{14}$ However, the low bioavailability of FLT after oral administration could be attributed to its poor wettability, low aqueous solubility, poor permeability, or low concentration at the absorption surface. ${ }^{15}$ Moreover, FLT undergoes a rapid first-pass hepatic metabolism after oral administration, resulting in a relatively short half-life of 5-6 hours. Reported FLT toxicity includes hepatotoxicity, nausea, and diarrhea. Thus, the pharmacokinetics and dosage characteristics (250 mg three times daily) of FLT make it a suitable candidate for the design of controlled-release delivery systems in order to enhance patients' compliance and to reduce the incidence of side effects. ${ }^{16}$

Various attempts have been made to improve FLT bioavailability and control its release behavior, including cyclodextrin inclusion complexes, ${ }^{15-17}$ colyophilized dispersions, ${ }^{18,19}$ liquisolid compacts, ${ }^{20}$ liposomes, ${ }^{21}$ nanoemulsions, ${ }^{22}$ and self-nanoemulsifying drug delivery systems. ${ }^{23}$ In our laboratory, ${ }^{24}$ oral controlled-release formulas of FLT were developed by combining immediate-release FLT-lyophilized monophase dispersions with prolonged-release FLT-chitosan microspheres. The developed formulas were found effective in providing a prolonged release of FLT for a long period of time after a suitable initial burst release. ${ }^{24}$

Commonly, nanoparticles composed of biodegradable polymers exhibit controlled release of their drug payload by diffusion or polymer degradation. These systems may provide prolonged exposure of the drug at their site of action once they have accumulated at their target. Nanoparticles have also been used to increase the aqueous solubility of several hydrophobic anticancer drugs via solubilization within the hydrophobic core of the nanoparticles, and to improve their in vivo performance, providing a repository for the drug to be slowly released. ${ }^{25}$ Therefore, another approach for improving FLT bioavailability and reducing its toxicity could be the formulation of biodegradable FLT-loaded nanoparticles for parenteral administration.

In this study we present the next generation of proteinbased nanoparticles prepared via ionic crosslinking, employing CAS as a matrix and sodium tripolyphosphate (TPP) as a polyanionic crosslinker for controlled delivery of FLT. TPP is a nontoxic polyanion that can interact with polycationic polymers via electrostatic forces to form ionic crosslinked networks. ${ }^{24}$ We suggest this novel system as a potential alternative to the chemically crosslinked protein nanoparticles, so as to avoid their possible toxicity. We attempted to encapsulate FLT into CAS nanoparticles to control its release rate, as well as extending its systemic circulation, comparing its pharmacokinetic parameters with those of drug cosolvent after intravenous administration in rats.

\section{Materials and methods Materials}

CAS from bovine milk, technical grade, and TPP, 90\%-95\%, practical grade, were purchased from Sigma-Aldrich (St Louis, MO, USA). FLT was kindly donated by Archimica (Origgio, Italy). Polyoxyethylene sorbitan monooleate (Tween 80) was from Riedel-de Häen (Seelze, Germany). Trypsin 2000 U/G from pancreas and sodium azide were obtained from LOBA Chemie Pvt, Ltd (Mumbai, India). Poly(ethylene glycol) 200 (PEG-200) was supplied by Pharaonia Pharmaceuticals (Alexandria, Egypt). Methylene chloride was obtained from ADWIC, El-Nasr Pharmaceutical Chemicals Co (Cairo, Egypt). All other chemicals were of analytical grade and used without further purification. 


\section{Preparation of ionically crosslinked FLT- loaded CAS nanoparticles}

Aqueous CAS solution (1\% w/v) was adjusted to $\mathrm{pH} 2.0$ with $1 \mathrm{~N}$ hydrochloric acid. Tween $80(2 \% \mathrm{v} / \mathrm{v})$ was added as a surfactant to CAS solution under magnetic stirring $(\mathrm{RH}$ basic, Ika Labortechnik, Staufen, Germany). Methylene chloride solution of FLT as the oil phase was mixed with aqueous CAS phase by homogenization (ULTRA-TURAX T25, IKA Labortechnik, Germany) at a speed of 13,000 rpm for 20 minutes to obtain an oil-in-water emulsion. The ratio of oil and aqueous phase was $1: 10 \mathrm{v} / \mathrm{v}$. TPP solution $(0.5 \%$ w/v) was added dropwise to the oil-in-water emulsion under gentle magnetic stirring. After 2 hours of crosslinking, nanoparticles were isolated by centrifugation (Sigma laboratory refrigerated centrifuge, model 3K-30, Germany) at 20,000 $\times \mathrm{g}$ and $10^{\circ} \mathrm{C}$ for 30 minutes, and subsequently washed several times with Tween 80 solution $(0.1 \% \mathrm{v} / \mathrm{v})$ and water. The particles were lyophilized (CRYODOS-50 Freeze-drier, Telstar Cryodos, Spain) and stored in dry conditions at $25^{\circ} \mathrm{C}$. The composition of CAS nanoparticles prepared with different formulation variables is shown in Table 1.

\section{Characterization of FLT-loaded CAS nanoparticles \\ Drug loading and incorporation efficiency}

The drug loading and incorporation efficiency of FLT-loaded CAS nanoparticles was determined by centrifugation of the colloidal samples at $20,000 \times \mathrm{g}$ and $4^{\circ} \mathrm{C}$ for 30 minutes. The nonentrapped FLT in the supernatant obtained after ultracentrifugation of nanoparticles was determined by high-performance liquid chromatography (HPLC) method. The amount of FLT entrapped within nanoparticles was calculated by the difference between the total amount used and the amount presented in the aqueous supernatant phase. All the experiments were carried out in triplicate. The percentage drug loading $(\% \mathrm{DL})$ and incorporation efficiency (\%IE) for each formulation were calculated using the following equations:

$$
\begin{aligned}
\% \mathrm{DL}= & (\text { Mass of drug in nanoparticles } / \text { mass of } \\
& \text { nanoparticles recovered }) \times 100
\end{aligned}
$$

A reverse-phase HPLC method was used for quantifying FLT. ${ }^{24}$ HPLC analysis was carried out with a PerkinElmer series 200 chromatograph (PerkinElmer, Waltham, MA, USA) using a Spheri-5, RP-18, $220 \mathrm{~mm} \times 4.6 \mathrm{~mm}, 5 \mu \mathrm{m}$, column, and a UV detector (PerkinElmer, Waltham, MA, USA). An isocratic solvent system consisting of 75:25 (v/v) methanol/water was used at a flow rate of $1 \mathrm{~mL} / \mathrm{min}$ and an injection volume of $20 \mu \mathrm{L}$, and the peaks were detected at $304 \mathrm{~nm}$. Under these experimental conditions, the total run time was approximately 6 minutes and the retention time was 3.9 minutes. Calibration curves (peak area versus[vs] drug concentration) were linear $\left(\mathrm{R}^{2}>0.999\right)$ over the FLT concentration range of $0.6-60 \mu \mathrm{g} / \mathrm{mL}$.

\section{Particle size and zeta $(\zeta)$ potential}

Particle size of FLT-loaded CAS nanoparticles was measured using a dynamic light scattering (DLS) technique with a NanoZS/ZEN3600 Zetasizer (Malvern, Instruments Ltd, Malvern, UK). This system is equipped with a $4 \mathrm{~mW}$ helium/neon laser at $633 \mathrm{~nm}$ wavelength and measures the

\begin{tabular}{|c|c|c|c|c|c|}
\hline Formula & Variable & DL $(\% w / w)$ & IE (\%w/w) & Particle size $(\mathrm{nm})$ & Zeta potential $(\mathrm{mV})$ \\
\hline & TPP:CAS & & & & \\
\hline$F_{1}$ & $1: 3$ & $2.50 \pm 0.02$ & $64.55 \pm 1.20$ & $100.60 \pm 3.66$ & $+7.54 \pm 0.04$ \\
\hline $\mathrm{F}_{2}$ & $\mathrm{I}: 5$ & $3.96 \pm 0.01$ & $44.09 \pm 1.58$ & $96.85 \pm 2.45$ & $+11.16 \pm 0.12$ \\
\hline $\mathrm{F}_{3}$ & $1: 10$ & $5.33 \pm 0.13$ & $36.31 \pm 0.73$ & $82.55 \pm 3.01$ & $+16.72 \pm 0.26$ \\
\hline \multirow[t]{2}{*}{$\mathrm{F}_{4}$} & $\mathrm{I}: 20$ & $7.72 \pm 0.06$ & $27.61 \pm 1.03$ & $64.83 \pm 2.87$ & $+17.30 \pm 0.15$ \\
\hline & FLT:CAS & & & & \\
\hline $\mathrm{F}_{5}$ & $1: 3$ & $8.73 \pm 0.04$ & $17.42 \pm 0.53$ & $61.90 \pm 2.10$ & $+15.47 \pm 0.30$ \\
\hline $\mathrm{F}_{6}$ & $\mathrm{I}: 5$ & $6.22 \pm 0.05$ & $30.46 \pm 0.72$ & $70.76 \pm 3.44$ & $+14.32 \pm 0.15$ \\
\hline \multirow[t]{2}{*}{$\mathrm{F}_{7}$} & $\mathrm{I}: 7$ & $5.28 \pm 0.12$ & $38.17 \pm 1.46$ & $86.84 \pm 3.03$ & $+|3.85 \pm 0.2|$ \\
\hline & CAS conc & & & & \\
\hline $\mathrm{F}_{8}$ & 2.5 & $3.53 \pm 0.04$ & $26.03 \pm 0.48$ & $75.22 \pm 2.76$ & $+15.10 \pm 0.26$ \\
\hline $\mathrm{F}_{9}$ & 5 & $3.76 \pm 0.05$ & $30.53 \pm 0.23$ & $83.24 \pm 3.91$ & $+14.63 \pm 0.33$ \\
\hline
\end{tabular}
particle size with the noninvasive backscattering technology at a detection angle of $173^{\circ}$ after an at least 100 -fold dilution

Table I Composition and characteristics of the formulated ionically crosslinked flutamide (FLT)-loaded casein (CAS) nanoparticles

Notes: Data expressed as mean \pm standard deviation, $n=3 .{ }^{a}{ }_{1-4}$ were prepared using $1 \%$ CAS and $\mathrm{I}: 10 \mathrm{FLT}$ :CAS mass ratio; ${ }^{b} \mathrm{~F}_{5-7}$ were prepared using $1 \%$ CAS and $\mathrm{I:5}$ TPP:CAS mass ratio; ${ }^{\circ} F_{8,9}$ were prepared using I:5 TPP:CAS and I:10 FLT:CAS mass ratios.

Abbreviations: DL, drug loading; IE, incorporation efficiency; TPP, sodium tripolyphosphate. 
with purified water. All of the DLS measurements were performed at $25.0^{\circ} \mathrm{C} \pm 0.1^{\circ} \mathrm{C}$ at 20 -second intervals for three repeat measurements. For the zeta potential measurement, each diluted nanoparticle suspension was put in a universal folded capillary cell equipped with platinum electrodes. The electrophoresis mobility was measured and the zeta potential was calculated by the Dispersion Technology Software provided by Malvern.

\section{Morphological analysis}

Transmission electron microscope (TEM) imaging of FLTloaded CAS nanoparticle suspension $\left(\mathrm{F}_{2}\right)$ was performed on a JEM-100 CX electron microscope (JEOL, Tokyo, Japan) at an accelerating voltage of $80 \mathrm{kV}$. After sample dilution with water (1:1000), a sample drop was placed on a copper grid. The excess was drawn off with a filter paper. Samples were subsequently stained with uranyl acetate solution for 30 seconds and then naturally dried.

\section{Differential scanning calorimetry}

To investigate the physical state of FLT inside CAS nanoparticles, thermograms of CAS, FLT, their respective physical mixture, and unloaded and FLT-loaded CAS nanoparticles $\left(\mathrm{F}_{2}\right)$ were recorded by differential scanning calorimetry model DSC 6 (PerkinElmer). Samples (2-4 mg) were placed in sealed aluminum pans and heated at $10^{\circ} \mathrm{C} / \mathrm{min}$ under nitrogen atmosphere (flow rate $20 \mathrm{~mL} / \mathrm{min}$ ) in the range of $30^{\circ} \mathrm{C}-300^{\circ} \mathrm{C}$.

\section{Fourier transform infrared spectra}

The Fourier transform infrared (IR) spectra of CAS, FLT, their respective physical mixture, and unloaded and FLTloaded CAS nanoparticles $\left(\mathrm{F}_{2}\right)$ were recorded using a Spectrum RXI Fourier transform IR spectrometer (PerkinElmer). Samples were finely ground with IR-grade $\mathrm{KBr}$ then pressed into pellets, and IR spectra were taken in transmission over the range of $4000-500 \mathrm{~cm}^{-1}$.

\section{Biodegradability of CAS nanoparticles}

To determine the degradation stability of the ionically crosslinked CAS nanoparticles, in vitro degradation was performed by digesting the nanoparticles in $\mathrm{pH} 7.4$ phosphate-buffered saline (PBS) containing trypsin. After centrifugation, the sedimented nanoparticles were redispersed in PBS ( $\mathrm{pH} 7.4$ ) by sonication. Trypsin ( $2 \mathrm{mg} / \mathrm{mL})$ was added to the nanoparticle suspension and the samples were incubated in a shaking water bath at $37^{\circ} \mathrm{C} \pm 0.5^{\circ} \mathrm{C}$ under mild horizontal shaking at $50 \mathrm{rpm}$ (GFL, type 1083, Gmbh and Co, Burgwedel, Germany). The turbidity of the nanoparticle suspension was monitored spectrophotometrically by recording the percent transmission at $600 \mathrm{~nm}$ at specified time intervals using T80 UV/VIS spectrophotometer (PG Instruments Ltd, London, UK).

\section{In vitro drug release}

In vitro release of FLT from the drug-loaded CAS nanoparticles was assessed using a dialysis bag method and compared with the drug solution in a cosolvent mixture of $0.9 \% \mathrm{w} / \mathrm{v} \mathrm{NaCl} /$ ethanol/PEG-200 (2:1:3 v/v/v) $\left(\mathrm{F}_{0}\right)$. FLT nanoparticles (equivalent to $20 \mathrm{mg}$ drug) were dispersed in PBS ( $\mathrm{pH}$ 7.4) and placed in a cellulose ester dialysis membrane, cut-off 12-14 kDa, sealed with appropriate universal closures (Spectrum Laboratories, Inc, Rancho Dominguez, CA, USA). The bags were tied to the paddle of the USP XXIV dissolution apparatus II, Type PTWS3 (Pharma Test, Hainburg, Germany), and dialyzed against $900 \mathrm{~mL}$ PBS ( $\mathrm{pH} 7.4$ ) containing $0.2 \%$ Tween 80 and $0.02 \%$ sodium azide as a preservative. The entire system was incubated at $37^{\circ} \mathrm{C} \pm 0.5^{\circ} \mathrm{C}$ under stirring at $100 \mathrm{rpm}$. At designated time intervals, $5 \mathrm{~mL}$ of the release medium was removed and replaced with the same volume of fresh PBS solution. All samples were run in triplicate and filtered through a $0.45 \mu \mathrm{m}$ membrane filter, and the amount of FLT released was analyzed by HPLC.

\section{In vivo pharmacokinetics}

In vivo experiments were performed on male Sprague Dawley rats $(200 \pm 20 \mathrm{~g})$ housed in stainless steel mesh cages in two groups of eight rats each, under standard conditions of light illumination, relative humidity, and temperature, and they had free access to standard laboratory food and water throughout the study. All procedures were performed according to a protocol approved by the Animal Care and Use Committee of the Faculty of Pharmacy, Alexandria University, Alexandria, Egypt, and in accordance with regulations of the National Research Council's guide for the care and use of laboratory animals.

For the pharmacokinetic studies, the ionically crosslinked FLT-loaded CAS nanoparticle formulation $\left(\mathrm{F}_{2}\right)$ was compared with the drug cosolvent $\left(\mathrm{F}_{0}\right) .{ }^{16}$ Rats were anesthetized with ether inhalation and injected intravenously via the tail vein with a single dose of FLT cosolvent or FLT-loaded CAS nanoparticles $(12 \mathrm{mg} / \mathrm{kg})$ per rat. Blood samples $(1 \mathrm{~mL})$ were collected from the retro-orbital plexus at designated time intervals (30 minutes, $1,2,4,6,8,12,24$, and 48 hours) in ethylenediaminetetraacetic acid-pretreated tubes. Samples were centrifuged immediately 
at $5000 \mathrm{rpm}$ for 10 minutes. The plasma samples were diluted to $2 \mathrm{~mL}$ with methanol, vortexed for 10 minutes, and then centrifuged at $8000 \mathrm{rpm}$ for 20 minutes. A $20 \mu \mathrm{L}$ amount of the supernatant was injected into the HPLC column to determine the FLT plasma concentration. The different pharmacokinetic parameters were calculated using noncompartmental methods with the software WinNonlin ${ }^{\circledR}$, Version 3.0 (Pharsight Corporation Ltd, Sunnyvale, CA, USA).

\section{Statistics}

All measurements were performed in triplicate, and the results were expressed as arithmetic mean \pm standard deviation.

\section{Results and discussion Formation of CAS nanoparticles}

Ionically crosslinked FLT-loaded CAS nanoparticles were successfully prepared by a two-step process. The first step involved the formation of oil droplets (encapsulating FLT) by an oil-in-water emulsion formation. Dichloromethane $\left(\mathrm{CH}_{2} \mathrm{Cl}_{2}\right)$ was chosen due to its ability to diffuse into the aqueous phase at a rapid rate, facilitating particle formation upon evaporation. ${ }^{26}$ The second step was the solidification of the formed droplets by ionic crosslinking of CAS ( $\mathrm{pH} 2)$ enveloping the oil droplets with TPP. By decreasing the $\mathrm{pH}$ of CAS solution below its isoelectric point (4.6-4.8), the amino groups of CAS become positively charged and can strongly attract the negatively charged phosphate groups of TPP, leading to the formation of ionically crosslinked nanoparticles. Figure 1 shows a photograph of uncrosslinked CAS $(1 \% \mathrm{w} / \mathrm{v})$ and CAS nanoparticles ionically crosslinked with two different concentrations of TPP (1:10 and 1:5 TPP:CAS ratios). It is clear that the visual turbidity of the nanoparticle suspension increases as the TPP concentration increases, indicating a higher crosslinking density.

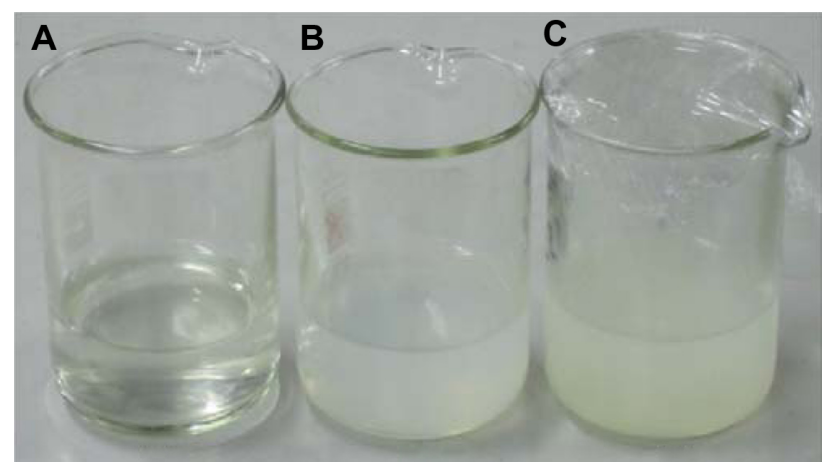

Figure I A photograph illustrating the difference in visual turbidity between: (A) casein (CAS) solution and ionically crosslinked CAS nanoparticles prepared with (B) I:10 and (C) 1:5 sodium tripolyphosphate (TPP):CAS mass ratios.
Few studies show the formation of protein nanoparticles via ionic interaction as an alternative to chemical crosslinking. The protein nature of CAS enables the formation of nanoparticles via electrostatic complexation with polyanionic polysaccharides, eg, gum Arabic, ${ }^{27}$ or polycationic polysaccharides, eg, chitosan, ${ }^{28}$ by adjusting the $\mathrm{pH}$ of CAS below or above its pI, respectively. In another study, Rediguieri et $\mathrm{al}^{29}$ investigated the electrostatic complexation between $\mathrm{CAS}$ and pectin. At $\mathrm{pH}>6$, pectin/CAS mixtures phase separate, as both polymers carry negative charges and so repel each other. However, below its pI, CAS molecules become positively charged, and therefore electrostatic attraction between CAS and the anionic pectin occurs, leading to the formation of microparticles.

\section{Characteristics of FLT-loaded CAS nanoparticles}

The drug incorporation efficiency obtained from the drug content analysis of nanoparticles ranged from $17.42 \%$ to $64.55 \%$, with a drug loading in the range of $2.50 \%-8.73 \%$, depending upon the formulation variables studied (Table 1). The effect of crosslinking density expressed as the TPP:CAS mass ratio on drug encapsulation was studied. Table 1 shows that FLT incorporation efficiency increased from $27.61 \%$ to $64.55 \%$ by increasing TPP concentration from 1:20 to $1: 3$ TPP:CAS mass ratio. A high amount of TPP causes a rise in the crosslinking density of CAS matrix, with a consequential hindrance of the drug escape to the continuous phase during solidification of emulsion droplets, which enhances the drug entrapment while reduced the drug loading. Similar results were obtained by Ajun et $\mathrm{al}^{26}$ where the probucol encapsulating efficiency into chitosan nanoparticles increased by increasing TPP concentration, whereas the drug loading decreased. It was also found that reducing FLT loading led to an enhancement of its incorporation efficiency, with a maximum incorporation (44.09\%) attained at an FLT:CAS ratio of 1:10 compared with $17.42 \%$ obtained for FLT:CAS ratio of $1: 3$.

\section{Particle size and zeta potential measurements}

DLS results revealed that FLT-loaded CAS nanoparticles exhibited a particle size in the range of 61.9-100.6 nm (Table 1) with a polydispersity of $0.37-0.59$, which could be correlated to the size and composition of CAS micelles (50-500 $\mathrm{nm}$ in diameter, $150 \mathrm{~nm}$ average diameter). CASs are mixtures of four phosphoproteins, $\alpha \mathrm{S} 1-, \alpha \mathrm{S} 2-, \beta$-, and $\kappa-\mathrm{CAS}$, with a molecular weight range of $19-25 \mathrm{kDa}$, thus 
producing heterogeneous nanoparticle size distribution. ${ }^{3,4}$ Shapira et $\mathrm{al}^{8}$ showed that paclitaxel- $\beta$-CAS and vinblastine$\beta$-CAS micelles had mean diameters of around $100 \mathrm{~nm}$ and $150 \mathrm{~nm}$, respectively, with mono or bimodal particle size distributions for both drugs. In another study conducted by Gaiani et al, ${ }^{30}$ the hydrodynamic diameter of the rehydrated spray-dried CAS powders was found to be around $210 \mathrm{~nm}$, which may correspond to CAS micelle size, and the suspension was too polydisperse.

Figure 2A reveals a bimodal size distribution of FLTloaded CAS nanoparticles $\left(\mathrm{F}_{2}\right)$. The first peak was obtained at $126.5 \mathrm{~nm}$ diameter, representing $91.6 \%$ of the particles. The second peak was at $10.19 \mathrm{~nm}$ diameter, apparently monomeric protein molecules, and represents $8.4 \%$ of the particles with a Z-average of $96.85 \pm 2.45$. Similar bimodal distributions were obtained for different nanoparticle formulations. From Table 1, it is also clear that increasing TPP concentration from 1:20 to 1:3 TPP:CAS ratio resulted in a corresponding increase in particle size from $64.83 \mathrm{~nm}$ to $100.60 \mathrm{~nm}$, respectively, showing the possibility of modifying particle size. Similar findings were reported by Ajun et al, ${ }^{26}$ where the size of chitosan nanoparticles increased by increasing TPP concentration.

Zeta potential measurements of ionically crosslinked FLT-loaded CAS nanoparticles are presented in Table 1. The nanoparticles were positively charged with a zeta potential range of $+7.54 \mathrm{mV}$ to $+17.30 \mathrm{mV}$. The pure positively charged uncrosslinked CAS exhibited a zeta potential of $+20.0 \mathrm{mV}$ (data not shown). This positive charge is a result of the net electrostatic charge on the CAS surface at $\mathrm{pH} 2.0$, ie, below its isoelectric point, where the CAS amino groups become positively charged. Upon CAS crosslinking with the polyanionic TPP, the zeta potential started to decrease to $+17.30 \mathrm{mV}$ at TPP:CAS ratio of 1:20 (Figure 2B). This figure revealed a bimodal zeta potential distribution where two populations of particles exist within this sample. About $73 \%$ of the particles have a zeta potential of $+20.7 \mathrm{mV}$, whereas $26.1 \%$ of the particles have a lower value of $+4.45 \mathrm{mV}$. As the TPP concentration increased to $1: 3$, the zeta potential of the nanoparticles decreased progressively to a value of $+7.54 \mathrm{mV}$.

The charge on the nanoparticles is quite low; however, steric stabilization is probably possible through the hydrophilic א-CAS "hairy" layer, which provides a charged and diffuse surface layer surrounding CAS micelle and stabilizes it through intermicellar electrostatic and steric repulsion, similar to a polyelectrolyte brush. ${ }^{3,4,40}$ Similar findings were observed by Ye et al, ${ }^{27}$ where a charge of $+15 \mathrm{mV}$ was exhibited by sodium caseinate-gum Arabic nanoparticles. The authors suggested that the presence of hydrophilic gum molecules on the outside of the caseinate aggregate may be enough to sterically stabilize the nanoparticles and consequently prevent their self-aggregation. ${ }^{27}$

The lyophilized ionically crosslinked CAS nanoparticles were readily dispersible in water, forming a colloidal turbid dispersion with a slight insignificant increase in both particle size and zeta potential after freeze-drying. No additives, eg, mannitol or trehalose, were needed for efficient lyophilization, suggesting the protein itself acts as a cryo-protectant. These findings are in agreement with the work of Bachar et $\mathrm{al}^{10}$ on celecoxib-loaded $\beta$-CAS micelles.

\section{Morphological analysis}

Figure 3A shows the TEM of ionically crosslinked FLTloaded CAS nanoparticle formulation $\left(\mathrm{F}_{2}\right)$. The particles were observed to have a spherical shape with a diameter around 40-50 nm smaller than that obtained from DLS measurements $(96.85 \mathrm{~nm})$. This size discrepancy has been previously reported by $\mathrm{Wu}$ et $\mathrm{al}^{32}$ where the size of chitosan/TPP nanoparticles loaded with ammonium glycyrrhizinate $(20-80 \mathrm{~nm})$, as revealed by TEM, was much smaller than that obtained by DLS ( $>120 \mathrm{~nm})$. The DLS method gives the hydrodynamic diameter, and the nanoparticles may shrink during the sample drying and preparation in TEM. The remarkable shrinkage indicates that the nanoparticles have a gel structure and can contain a lot of water, which corroborates with the high water-binding capacity of CAS. ${ }^{31}$

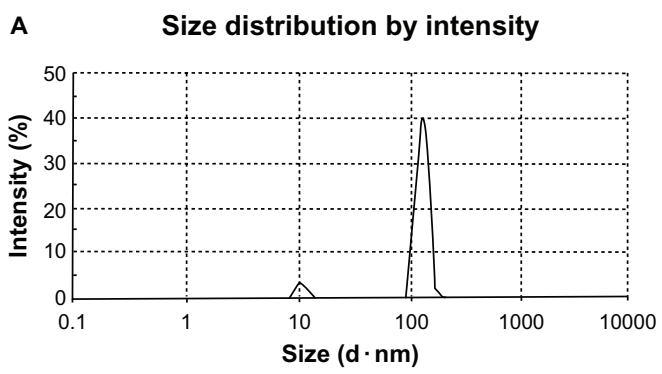

B

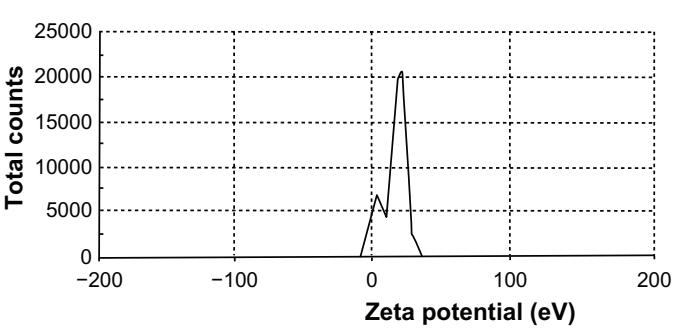

Figure 2 Particle size $(\mathbf{A})$ and zeta potential $(\mathbf{B})$ distributions of flutamide-loaded casein nanoparticles $\left(\mathrm{F}_{2}\right)$. 

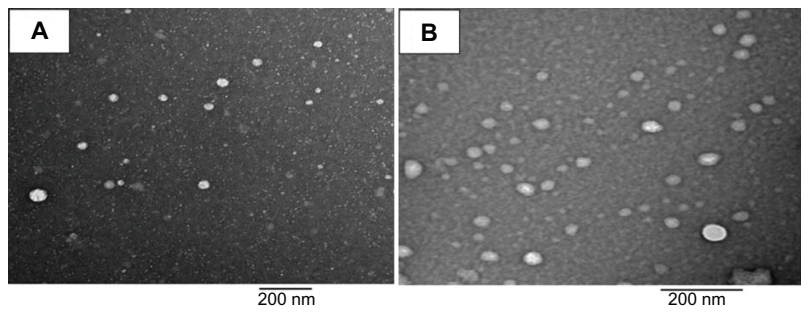

Figure 3 Transmission electron micrographs of flutamide-loaded casein nanoparticles $\left(F_{2}\right)$ at a magnification of $20,000 \times(\mathbf{A})$ and $30,000 \times(B)$.

A higher magnification illustrates to a certain extent that the nanoparticles may retain the core shell structure of CAS micelles (Figure 3B). From the micrographs, it is clear that the aggregation of CAS nanoparticles was prevented effectively maybe via the stabilizing effect of hydrophilic shells of $\kappa-C A S$.

\section{Solid state characterization}

The physical status of FLT formulated in the ionically crosslinked CAS nanoparticles was compared with the free drug using DSC (Figure 4). A CAS thermogram displayed a broad endothermic peak at $94.8^{\circ} \mathrm{C}$ due to the evolution of water from the sample, and another one at $205.6^{\circ} \mathrm{C}$. FLT in its natural state exists as crystals, which are characterized by the high melting peak around $112.8^{\circ} \mathrm{C} .{ }^{24}$ In the CAS/FLT physical mixture, the endothermic peaks of both FLT and CAS were detectable at their original positions. However, when
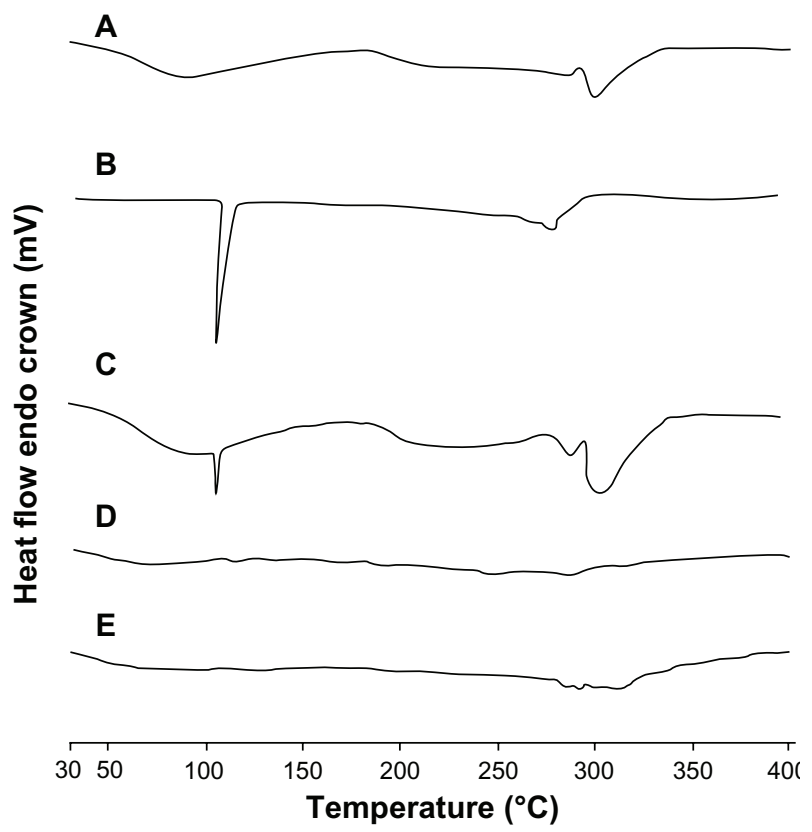

Figure 4 Differential scanning calorimetry thermograms of casein (CAS) (A), flutamide (FLT) (B), CAS-FLT physical mixture (C), unloaded CAS nanoparticles (D), and FLT-loaded CAS nanoparticles $\left(F_{2}\right)(E)$. the drug was formulated in CAS nanoparticles, the peak at its original melting point disappeared, and the thermogram was essentially similar to that of unloaded CAS nanoparticles. This may be explained by the total incorporation of the drug into the nanoparticles, suggesting a molecular dispersion of drug inside the protein matrix. Similar results were observed by Puthli and Vavia ${ }^{33}$ for levonorgestrel-loaded CAS microparticles.

In order to further characterize possible interactions between the drug and the protein carrier in the solid state, IR spectra of CAS, FLT, TPP, CAS-FLT physical mixture, and ionically crosslinked drug-loaded CAS nanoparticles were recorded (Figure 5). CAS typically shows absorption bands at $3455.86,1661.12,1530.51$, and $1235.4 \mathrm{~cm}^{-1}$ that originate from $\mathrm{N}-\mathrm{H}$ stretching and amide bending vibrations. CAS exhibited another characteristic band at $1415.9 \mathrm{~cm}^{-1}$, attributable to the carboxylate group $(\mathrm{O}-\mathrm{C}-\mathrm{O})$. FLT has a characteristic peak at $3358.3 \mathrm{~cm}^{-1}$, corresponding to $\mathrm{NH}$ stretching vibration of its secondary amino group in addition to the carbonyl stretching peak at $1715.7 \mathrm{~cm}^{-1}(\mathrm{C}=\mathrm{O}$ amide $) .{ }^{17-19}$

All the characteristic peaks of FLT are present in the physical mixture in their original positions. However, in the spectrum of FLT-loaded CAS nanoparticles, the characteristic peak of FLT at $3358.3 \mathrm{~cm}^{-1}$,corresponding to its amino group, was overlapped with the $\mathrm{N}-\mathrm{H}$ stretching vibration of CAS at $3455 \mathrm{~cm}^{-1}$. Furthermore, the carbonyl stretching peak of FLT at $1715.7 \mathrm{~cm}^{-1}$ was shifted to $1730.2 \mathrm{~cm}^{-1}$ with much reduced intensity. This result indicated a change in the environment of the carbonyl group of the drug as a consequence of a weak interaction (intermolecular hydrogen bonds) between the drug and protein during the nanoencapsulation process. ${ }^{17-19}$

From the IR spectrum of TPP, the stretching vibration of the $\mathrm{P}=\mathrm{O}$ or $\mathrm{P}-\mathrm{O}$ at $1217.1 \mathrm{~cm}^{-1}$ and $1157.9 \mathrm{~cm}^{-1}$ was observed. On comparing the IR spectrum of TPP-crosslinked CAS nanoparticles with the spectrum of TPP, some peaks disappeared due to interaction among groups of CAS and TPP. The amide bending vibration of CAS at $1235.7 \mathrm{~cm}^{-1}$ and the stretching vibration of the $\mathrm{P}=\mathrm{O}$ or $\mathrm{P}-\mathrm{O}$ of TPP at $1217.1 \mathrm{~cm}^{-1}$ disappeared, and a new peak was observed at $1245.7 \mathrm{~cm}^{-1}$ and $1243.4 \mathrm{~cm}^{-1}$ in the spectra of unloaded and FLT-loaded TPP-crosslinked CAS nanoparticles, respectively.

Furthermore, the IR spectrum of the TPP-crosslinked CAS nanoparticles exhibited a new peak at $3270.3 \mathrm{~cm}^{-1}$, indicating that the amino group of CAS may be involved in a bond formation with the phosphate group of TPP, and implying the complex formation via electrostatic interaction between phosphoric groups of TPP and ammonium ions 


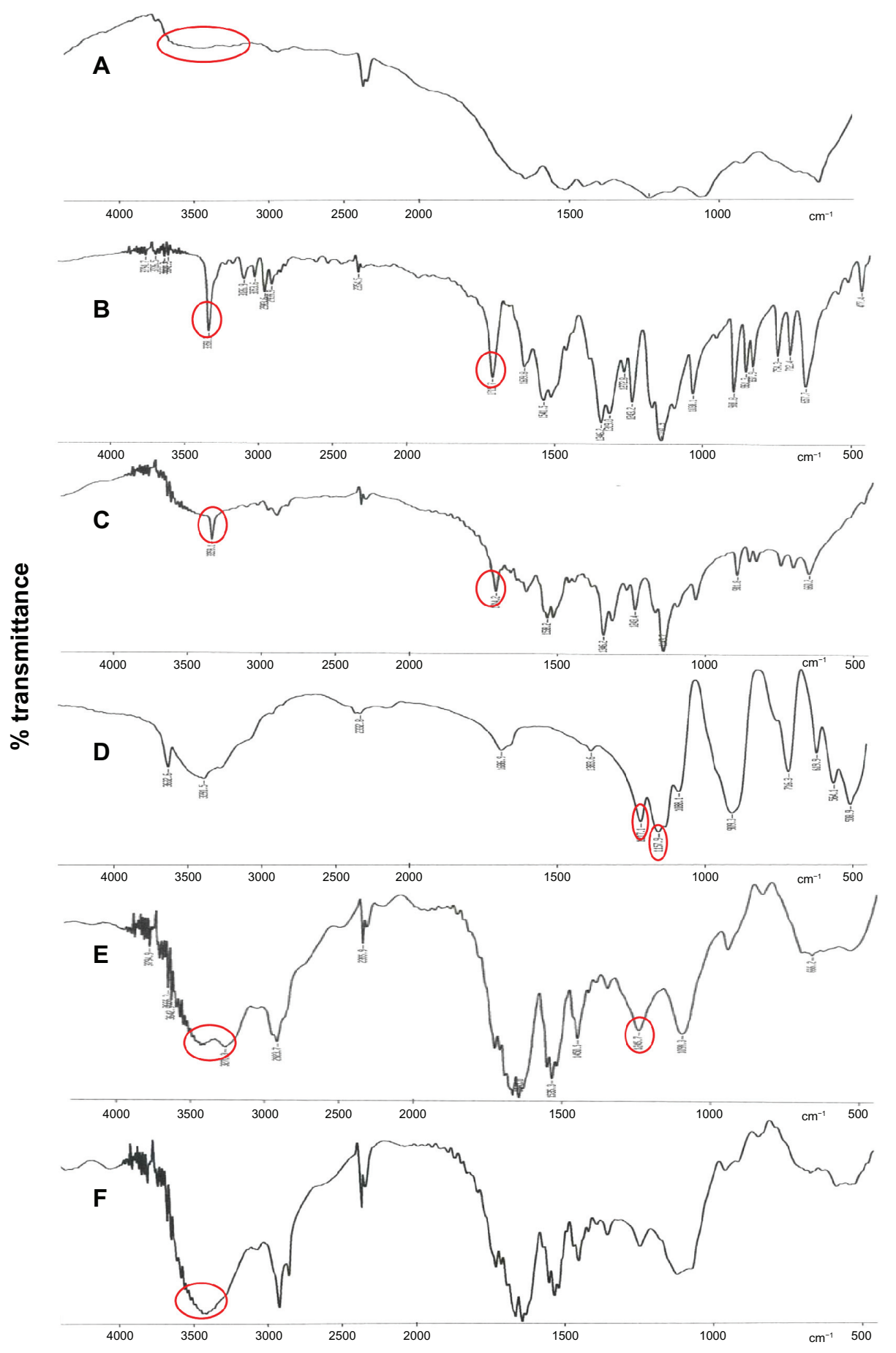

Figure 5 Fourier transform infrared spectroscopy transmission spectra of casein (CAS) (A), flutamide (FLT) (B), CAS-FLT physical mixture (C), sodium tripolyphosphate (D), unloaded CAS nanoparticles $(\mathbf{E})$, and FLT-loaded CAS nanoparticles $\left(F_{2}\right)(\mathbf{F})$.

Note: Red circles indicate characteristic peaks.

of CAS. At the same time, the $3415.8 \mathrm{~cm}^{-1}$ peak of CAS remains, indicating that not all the amino groups of CAS are involved in the reaction.

\section{Biodegradability of CAS nanoparticles}

Several factors, such as particle preparation technique, degradation environments, enzyme activity, surface area, porosity, tortuosity, and size, can affect the degradation of the matrix of protein nanoparticles. At neutral $\mathrm{pH}$, trypsin is expected to attack specific sites on the surface and in the interior of the protein particles. The development of a dense crosslinking matrix for nanoparticles offers resistance against the proteolytic degradation, as it is difficult for the enzymes to penetrate into the particles. ${ }^{34}$ 
In our study, proteolysis of ionically crosslinked CAS nanoparticles was measured by a turbidometric method. Figure 6 shows the percentage transmittance values at $600 \mathrm{~nm}$, obtained after digesting CAS nanoparticles with three different crosslinking densities in trypsin. It is clear that the ease with which CAS nanoparticles were degraded by the enzyme trypsin depended on the crosslinking density of the protein. Only $58.47 \%$ transmittance was achieved after incubation of CAS nanoparticles with a high crosslinking density (1:3 TPP:CAS mass ratio) in trypsin solution for 96 hours, whereas CAS nanoparticles with a low crosslinking density (1:10 TPP:CAS mass ratio) were completely degraded (98.81\% transmittance) after only 72 hours incubation in trypsin solution. As can be seen, the lower the crosslinking density, the faster the rate of degradation of the protein matrix and hence an increase in the percentage transmittance. This suggests that the residence time of nanoparticles in tissue or blood might be controlled by changing the crosslinking density of the CAS matrix. ${ }^{35}$

Similar results were obtained by Jayakrishnan et al ${ }^{35}$ where the protease degradation of CAS microspheres crosslinked with a higher amount of glutaraldehyde was slower than that crosslinked with a lower amount. In another study, Gunasekaran et $\mathrm{al}^{34}$ attributed the resistance of the glutaraldehyde-crosslinked $\beta$-lactoglobulin ( $\beta \mathrm{LG}$ ) nanoparticles against enzymatic attack to their dense crosslinked structure and small portion of basic amino acid composition.

\section{In vitro release of FLT from CAS nanoparticles}

A sustained-release pattern is a key issue in the development of colloidal drug delivery systems used in the field of nanomedicine. Ionically crosslinked FLT-loaded CAS nanoparticles prepared under experimental conditions described previously were tested in vitro and released at $37^{\circ} \mathrm{C}$ in PBS (pH 7.4) containing $0.2 \%$ Tween 80 over 96 hours. In general, FLT was released from the CAS nanoparticles very slowly in a continuous way for up to 4 days, showing an almost sustained-release ability of the nanoparticle formulations. On the contrary, it was found that $>80 \%$ of FLT cosolvent was released within the first 2 hours.

The crosslinking of protein nanoparticles is important for sustained release and targeted drug delivery. From Figure 7, it can be seen that the higher the crosslinking density, the slower the rate of drug release from the nanoparticles. After 96 hours, $91.54 \%$ of FLT was released from nanoparticles crosslinked with a 1:10 TPP:CAS mass ratio, compared with $74.23 \%$ from nanoparticles crosslinked with a 1:3 TPP:CAS mass ratio. The nanoparticles prepared with a lower TPP concentration showed a greater overall release. This may be attributed to reduced swelling and drug diffusion from CAS nanoparticles with the increased crosslinking density of the matrices owing to the increasing barrier for drug diffusion by the additional crosslinks formed at higher concentrations of TPP. Thus, it is possible to modulate the release of

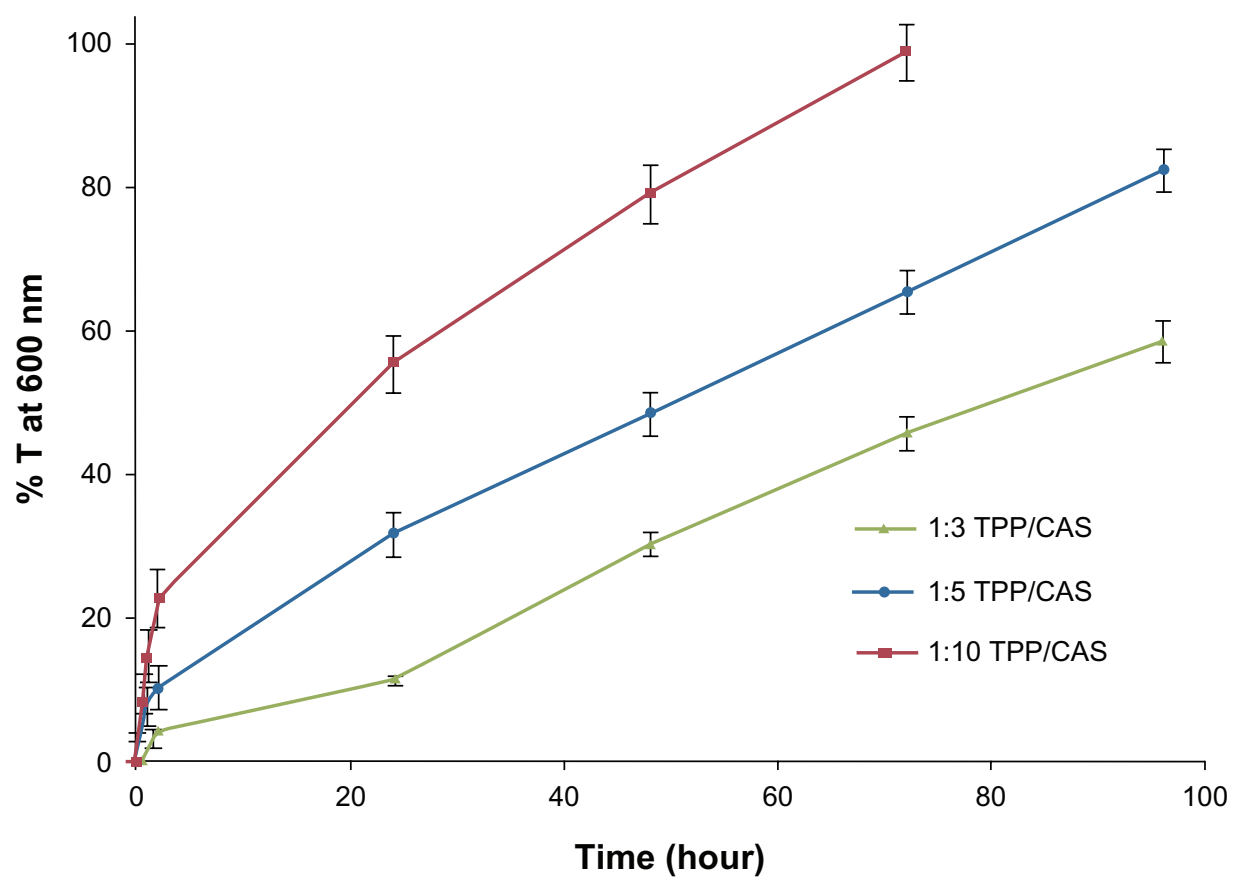

Figure 6 Influence of sodium tripolyphosphate (TPP) crosslinking density on the biodegradability of casein (CAS) nanoparticles in trypsin solution measured as \% transmittance (T) at $600 \mathrm{~nm}$. 


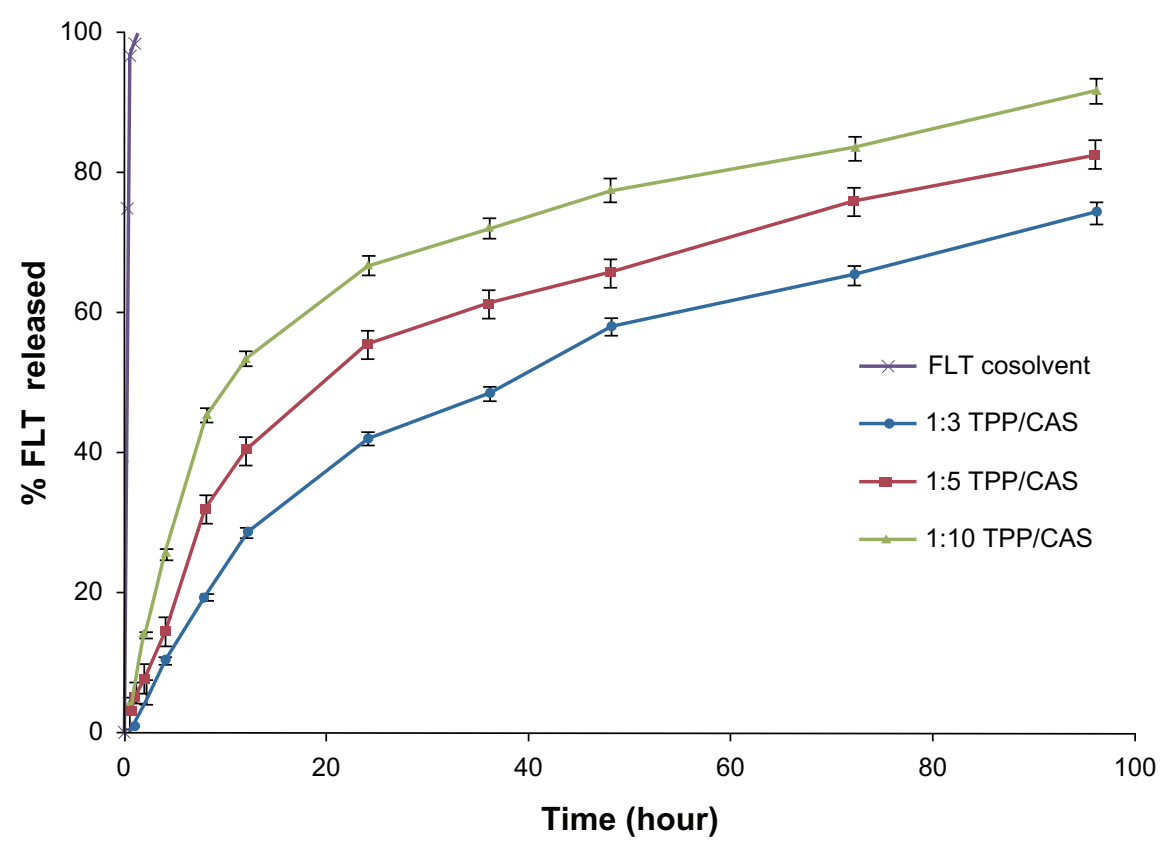

Figure 7 The influence of sodium tripolyphosphate (TPP) crosslinking density on flutamide (FLT) release from casein (CAS) nanoparticles in phosphate-buffered saline $(\mathrm{pH} 7.4)$ at $37^{\circ} \mathrm{C}$.

drugs from the protein matrix by changing the crosslinking density. These data suggest that combining drugs with CAS nanoparticles could prevent release at the injection site and enable the drug to be released slowly in order to sufficiently accumulate at the target site of action.

In a previous work, ascorbyl palmitate was more easily released from TPP-crosslinked chitosan nanoparticles at low TPP concentration because of the low-density structure. ${ }^{36}$ Previous studies showed a sustained-release ability of drugs (eg, mitoxantrone ${ }^{37}$ and progesterone ${ }^{38}$ ) from glutaraldehyde-crosslinked CAS microparticles, where the rate of drug release was found to decrease with the increase in the glutaraldehyde concentration.

\section{In vivo pharmacokinetics}

The mean plasma concentration over time for the FLT cosolvent $\left(\mathrm{F}_{0}\right)$ and FLT-loaded CAS nanoparticles $\left(\mathrm{F}_{2}\right)$ after intravenous administration into healthy rats is illustrated in Figure 8. FLT level in plasma decreased much more rapidly in the case of the FLT cosolvent system $\left(\mathrm{F}_{0}\right)$ when

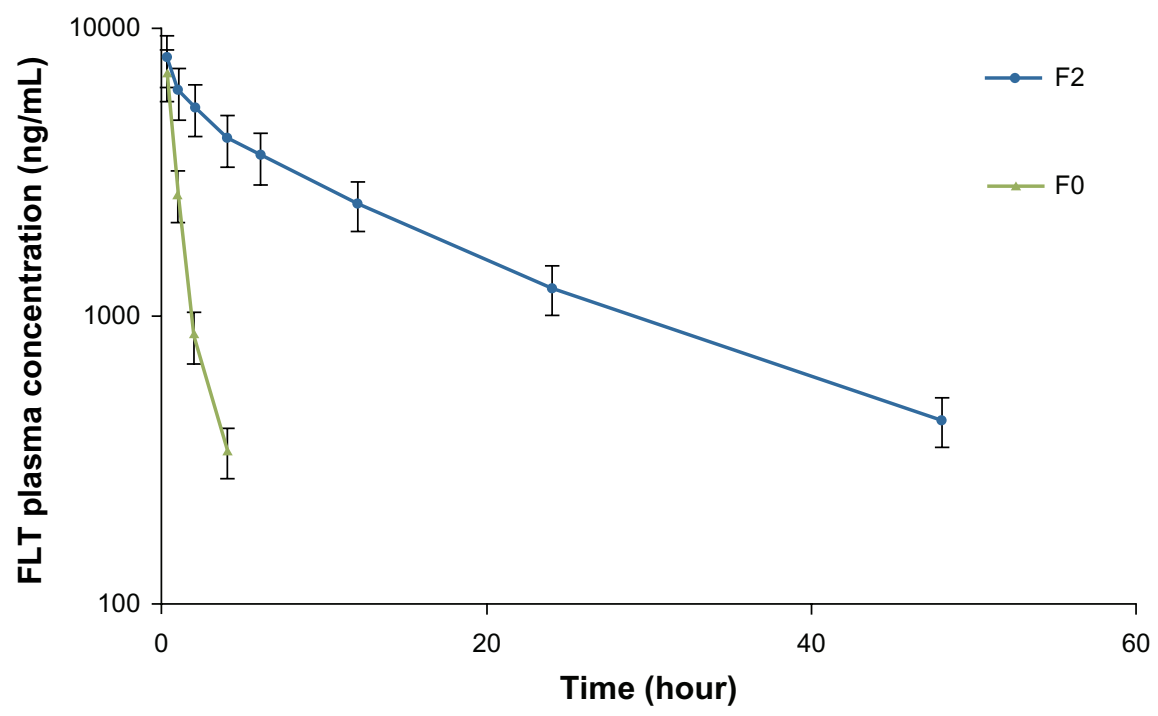

Figure 8 Plasma concentration of flutamide (FLT) following intravenous administration of a single dose of FLT cosolvent ( $\left.\mathrm{F}_{0}\right)$ and ionically crosslinked FLT-loaded casein nanoparticles $\left(F_{2}\right)(12 \mathrm{mg} / \mathrm{kg})$ into healthy rats. 
Table 2 Pharmacokinetic parameters of flutamide (FLT) after intravenous administration of a single dose of FLT cosolvent $\left(\mathrm{F}_{0}\right)$ and ionically crosslinked FLT-loaded casein nanoparticles $\left(\mathrm{F}_{2}\right)(12 \mathrm{mg} / \mathrm{kg})$ into healthy rats

\begin{tabular}{|c|c|c|c|c|c|c|c|c|}
\hline Formula & $T_{1 / 2}(h)$ & $\begin{array}{l}C_{\max } \\
(\mu \mathrm{g} / \mathrm{mL})\end{array}$ & $\mathbf{T}_{\max }(\mathbf{h})$ & $\begin{array}{l}A U C_{i n f} \\
(\mu g \cdot h / m L)\end{array}$ & $\operatorname{MRT}_{\text {inf }}(h)$ & $\mathbf{K}_{\mathrm{el}}\left(\mathbf{h}^{-1}\right)$ & $V_{d}(L / k g)$ & $\begin{array}{l}\mathrm{CL} \\
(\mathrm{L} / \mathrm{h} \cdot \mathrm{kg})\end{array}$ \\
\hline $\mathrm{F}_{0}$ & $0.88 \pm 0.02$ & $7.10 \pm 0.5$ & $0.25 \pm 0.02$ & $7.97 \pm 0.22$ & $1.19 \pm 0.2$ & $0.65 \pm 0.1$ & $2.31 \pm 0.1$ & $1.51 \pm 0.2$ \\
\hline $\mathrm{F}_{2}$ & $14.64 \pm 1.5$ & $8.02 \pm 0.3$ & $0.39 \pm 0.03$ & $99.89 \pm 3.43$ & $18.52 \pm 0.7$ & $0.04 \pm 0.0$ & $2.76 \pm 0.2$ & $0.12 \pm 0.0$ \\
\hline
\end{tabular}

Note: Data expressed as mean \pm standard deviation, $\mathrm{n}=3$.

Abbreviations: $\mathrm{AUC}_{\text {inf }}$, the area under the plasma concentration-time curve; $\mathrm{CL}$, body clearance; $\mathrm{C}_{\max }$, the peak concentration in plasma; $\mathrm{h}$, hour; $\mathrm{K}_{\mathrm{el}}$, elimination rate constant; L, liter; $\mathrm{MRT}_{\text {inf }}$ mean residence time; $\mathrm{T}_{1 / 2}$, elimination half-life; $\mathrm{T}_{\max }$, the time to reach maximum concentration; $\mathrm{V}_{\mathrm{d}}$, volume of distribution.

compared with FLT-CAS nanoparticles. Clearly, FLT-loaded CAS nanoparticles exhibited a longer circulation time and a markedly delayed blood clearance with a drug level of $440.35 \mathrm{ng} / \mathrm{mL}$ at 48 hours after administration. On the contrary, drug cosolvent was quickly removed from the circulating system after administration, with a plasma concentration of about $343.45 \mathrm{ng} / \mathrm{mL}$ after only 4 hours.

The pharmacokinetic parameters determined through the statistical analysis showed that FLT-loaded CAS nanoparticles $\left(\mathrm{F}_{2}\right)$ could extend the half-life of FLT from 0.88 hours to 14.64 hours (Table 2). Meanwhile, the area under the FLT concentration-time curve $\left(\mathrm{AUC}_{\text {inf }}\right)$ increased by about 12.5 -fold for FLT nanoparticles $\left(\mathrm{F}_{2}\right)$ compared with FLT cosolvent $\left(\mathrm{F}_{0}\right)$. There was an inverse relationship between the nanocarrier clearance by the reticuloendothelial system (RES) and their prolonged circulation time. The mean clearance value of FLT cosolvent $\left(\mathrm{F}_{0}\right)$ was 12.6-fold greater than FLT nanoparticles $\left(\mathrm{F}_{2}\right)$. Therefore, it appears that the longer half-life and pronounced increase in the blood residence time of FLT-loaded CAS nanoparticles was the result of a reduced clearance rate.

It has been found that particles under $200 \mathrm{~nm}$ in diameter display a decreased rate of clearance and thus an extended circulation time. In addition, proteins have the possibility of less opsonization by RES through an aqueous steric barrier. ${ }^{2}$ Therefore, the prolonged circulation of CAS nanoparticles may be due to their ability to reduce the uptake of FLT by the RES, which may reduce side effects associated with the hepatic system. The hydrophobic core of CAS nanoparticles could also retain their drug content in plasma for a longer time. ${ }^{11,25}$ Based on the findings of the pharmacokinetic study, we postulate that the hydrophilic $\kappa$-CAS layer covering the surface of CAS micelles can simulate the role of PEG hydrophilic shellsuppressing opsonization through generating a steric barrier, preventing hydrophobic interactions of plasma opsonins with the particle surface and inhibiting the uptake by RES. ${ }^{39}$

\section{Conclusions}

In this work we investigated a new kind of colloidal system, ionically crosslinked CAS nanoparticles, as a vehicle for effective solubilization and controlled delivery of the poorly soluble antiandrogen FLT. Our results demonstrated that CAS nanoparticles are promising candidates for controlled drug delivery, as they can be easily prepared under mild conditions and they can incorporate bioactive compounds with reasonable entrapment efficiency. Moreover, they presented small particle size (below $100 \mathrm{~nm}$ ), positive zeta potential, and a good redispersibility after lyophilization. The nanoparticles succeeded in achieving a sustained-release pattern of the drug with the release rate and could be modified via modulating the crosslinking density. In vivo assessment of FLT-loaded CAS nanocarriers demonstrated that they were well tolerated in vivo and caused a significant prolongation of FLT circulation in plasma, compared with drug cosolvent. These properties are extremely useful for intravenous delivery of hydrophobic anticancer drugs that are poorly absorbed.

\section{Acknowledgment}

The authors thank Archimica Chemical Company, Italy, for kind donation of the FLT used in this study.

\section{Disclosure}

The authors report no conflicts of interest in this work.

\section{References}

1. Chen L, Remondetto GE, Subirade M. Food protein-based materials as nutraceutical delivery systems. Trends Food Sci Technol. 2006;17: 272-283.

2. Elzoghby AO, Samy WM, Elgindy NA. Protein-based nanocarriers as promising drug and gene delivery systems. J. Control Release. 2012;161: 38-49.

3. Livney YD. Milk proteins as vehicles for bioactives. Curr Opin Colloid Interface Sci. 2010;15:73-83.

4. Elzoghby AO, Abo El-Fotoh WS, Elgindy NA. Casein-based formulations as promising controlled release drug delivery systems. J Control Release. 2011;153:206-216.

5. Semo E, Kesselman E, Danino D, Livney YD. Casein micelle as a natural nanocapsular vehicle for nutraceuticals. Food Hydrocoll. 2007;21: 936-942.

6. Zimet P, Rosenberg D, Livney YD. Re-assembled casein micelles and casein nanoparticles as nano-vehicles for $\omega-3$ polyunsaturated fatty acids. Food Hydrocoll. 2011;25:1270-1276.

7. Esmaili M, Ghaffari SM, Moosavi-Movahedi Z, et al. Beta casein-micelle as a nano vehicle for solubility enhancement of curcumin; Food industry application. LWT Food Sci Technol. 2011;44:2166-2172. 
8. Shapira A, AssarafYG, Epstein D, Livney YD. $\beta$-casein nanoparticles as an oral delivery system for chemotherapeutic drugs: Impact of drug structure and properties on co-assembly. Pharm Res. 2010;27: 2175-2186.

9. Shapira A, Davidson I, Avni N, Assaraf YG, Livney YD. $\beta$-Casein nanoparticle-based oral drug delivery system for potential treatment of gastric carcinoma: Stability, target-activated release and cytotoxicity. Eur J Pharm Biopharm. 2012;80:298-305.

10. Bachar M, Mandelbaum A, Portnaya I, Perlstein, et al. Development and characterization of a novel drug nanocarrier for oral delivery, based on self-assembled $\beta$-casein micelles. J Control Release. 2012;160: 164-171.

11. Elzoghby AO, Samy WM, Elgindy NA. Albumin-based nanoparticles as potential controlled release drug delivery systems. J Control Release. 2012;167:168-182.

12. Elzoghby AO, Samy WM, Elgindy NA. Novel spray-dried genipincrosslinked casein nanoparticles for prolonged release of alfuzosin hydrochloride. Pharm Res. 2013;30:512-522.

13. Martel CL, Gumerlock PH, Meyers FJ, Lara PN. Current strategies in the management of hormone refractory prostate cancer. Cancer Treat Rev. 2003;29:171-187.

14. Goldspiel BR, Kohler DR. Flutamide: an antiandrogen for advanced prostate cancer. DICP. 1990;24:616-623.

15. Zuo Z, kwon G, Stevenson B, Diakur J, Wiebe LI. Hydroxypropyl$\beta$-cyclodextrin-flutamide inclusion complex. I. Formulation, physical characterization and absorption studies using the Caco-2 in vitro model. J Pharm Pharmaceut Sci. 2000;3:220-227.

16. Zuo Z, Tam YK, Diakur J, Wiebe LI. Hydroxypropyl- $\beta$-cyclodextrin flutamide inclusion complex. II. Oral and intravenous pharmacokinetics of flutamide in rat. J Pharm Pharmaceut Sci. 2002;5:292-298.

17. Elgindy N, Elkhodairy K, Molokhia A, Elzoghby A. Lyophilization monophase solution technique for improvement of the physicochemical properties of an anticancer drug. flutamide. Eur J Pharm Biopharm. 2010;74:397-405.

18. Elgindy N, Elkhodairy K, Molokhia A, Elzoghby A. Lyophilization monophase solution technique for preparation of amorphous flutamide dispersions. Drug Dev Ind Pharm. 2011;37:446-455.

19. Elgindy N, Elkhodairy K, Molokhia A, Elzoghby A. Lyophilized flutamide dispersions with polyols and amino acids: preparation and in vitro evaluation. Drug Dev Ind Pharm. 2011;37:446-455.

20. Elkhodairy K, Samy W. Optimization and evaluation of micromeritic and release properties of high dose flutamide liquisolid systems. Lett Drug Des Discov. 2012;9:336-344.

21. Murthy RSR, Umrethia ML. Optimization of formulation parameters for the preparation of flutamide liposomes by 3(3) factorial 26-term logit model. Pharm Dev Technol. 2004;9:369-377.

22. Madhusudhan B, Rambhau D, Apte SS, Gopinath D. Oral bioavailability of flutamide from 1-O-alkylglycerol stabilized o/w nanoemulsions. J Disp Sci Technol. 2007;28:1254-1261.
23. Jeevana JB, Sreelakshmi K. Design and evaluation of self-nanoemulsifying drug delivery system of flutamide. J Young Pharm. 2011;3:4-8.

24. Elgindy N, Elkhodairy K, Molokhia A, Elzoghby A. Biopolymeric microparticles combined with lyophilized monophase dispersions for controlled flutamide release. Int J Pharm. 2011;411:113-120.

25. Kumari A, Yadav SK, Yadav SC. Biodegradable polymeric nanoparticles based drug delivery systems. Colloids Surf B Biointerfaces. 2010;75: $1-18$.

26. Ajun W, Yan S, Li G, Huili L. Preparation of aspirin and probucol in combination loaded chitosan nanoparticles and in vitro release study. Carbohydr Polym. 2009;75:566-574.

27. Ye A, Flanagan J, Singh H. Formation of stable nanoparticles via electrostatic complexation between sodium caseinate and gum Arabic. Biopolym. 2006;82:121-133.

28. Anal AK, Tobiassen A, Flanagan J, Singh H. Preparation and characterization of nanoparticles formed by chitosan-caseinate interactions. Colloids Surf B Biointerfaces. 2008;64:104-110.

29. Rediguieri CF, de Freitas O, Lettinga MP, Tuinier R. Thermodynamic incompatibility and complex formation in pectin/caseinate mixtures. Biomacromolecules. 2007;8:3345-3354.

30. Gaiani C, Mullet M, Arab-Tehrany E, et al. Milk proteins differentiation and competitive adsorption during spray-drying. Food Hydrocoll. 2011;25:983-990.

31. Pan X, Yu S, Yao P, Shao Z. Self-assembly of $\beta$-casein and lysozyme. J Colloid Interf Sci. 2007;316:405-412.

32. Wu Y, Yang W, Wang C, Hu J, Shoukuan F. Chitosan nanoparticles as a novel delivery system for ammonium glycyrrhizinate. Int $J$ Pharm. 2005;295:235-245.

33. Puthli S, Vavia P. Gamma irradiated micro system for long-term parenteral contraception: an alternative to synthetic polymers. Eur J Pharm Sci. 2008;35:307-317.

34. Gunasekaran S, Ko S, Xiao L. Use of whey proteins for encapsulation and controlled delivery applications. J Food Eng. 2007;83:31-40.

35. Jayakrishnan A, Knepp WA, Goldberg EP. Casein microspheres: preparation and evaluation as a carrier for controlled drug delivery. Int J Pharm. 1994;106:221-228.

36. Yoksana R, Jirawutthiwongchai J, Arpo K. Encapsulation of ascorbyl palmitate in chitosan nanoparticles by oil-in-water emulsion and ionic gelation processes. Colloids Surf B Biointerfaces. 2010;76:292-297.

37. Knepp WA, Jayakrishnan A, Quigg JM, Sitren HS, Bagnall JJ, Goldberg EP. Synthesis, properties, and intratumoral evaluation of mitoxantroneloaded casein microspheres in Lewis lung carcinoma. J Pharm Pharmacol. 1993;45:887-891.

38. Latha MS, Lal AV, Kumary TV, Sreekumar R, Jayakrishnan A. Progesterone release from glutaraldehyde cross-linked casein microspheres: in vitro studies and in vivo response in rabbits. Contraception. 2000;61:329-334.

39. De Kruif CG, Zhulina EB. K-Casein as a polyelectrolyte brush on the surface of casein micelles. Colloids Surf A. 1996;117:151-159.
International Journal of Nanomedicine

\section{Publish your work in this journal}

The International Journal of Nanomedicine is an international, peerreviewed journal focusing on the application of nanotechnology in diagnostics, therapeutics, and drug delivery systems throughout the biomedical field. This journal is indexed on PubMed Central, MedLine, CAS, SciSearch ${ }^{\circledR}$, Current Contents ${ }^{\circledR} /$ Clinical Medicine,

\section{Dovepress}

Journal Citation Reports/Science Edition, EMBase, Scopus and the Elsevier Bibliographic databases. The manuscript management system is completely online and includes a very quick and fair peer-review system, which is all easy to use. Visit http://www.dovepress.com/ testimonials.php to read real quotes from published authors. 